\title{
A Platelet Acquired Storage Pool Disorder Associated with Tamoxifen Therapy
}

\author{
Lalitha Nayak and Alvin H. Schmaier \\ Seidman Cancer Center, University Hospitals Case Medical Center and Division of Hematology and Oncology, \\ Department of Medicine, Case Western Reserve University, 10900 Euclid Avenue, WRB2-130, Cleveland, OH 44106-7284, USA \\ Correspondence should be addressed to Lalitha Nayak, lalitha.nayak@case.edu
}

Received 20 November 2012; Accepted 6 December 2012

Academic Editors: H. Guglielmone, M.-C. Kyrtsonis, Y. Matsukawa, and Y. Shiozawa

Copyright (๑) 2012 L. Nayak and A. H. Schmaier. This is an open access article distributed under the Creative Commons Attribution License, which permits unrestricted use, distribution, and reproduction in any medium, provided the original work is properly cited.

The antiestrogenic drug tamoxifen, used in patients with breast cancer, is associated with an increase in arterial and venous thrombotic events, the mechanism of which is not clearly understood. We report a case of a lady who presented with new bruising and prolonged bleeding following a tooth extraction 4-6 weeks after starting tamoxifen. Investigations were consistent with an acquired platelet storage pool disorder. Repeat platelet function analysis was normal, performed 3 months after discontinuation of tamoxifen. We present a previously clinically unreported effect of tamoxifen on platelet function.

\section{Introduction}

Tamoxifen, an antiestrogenic drug belonging to the selective estrogen receptor modulator (SERM), is widely used for the prevention and treatment of breast cancer. It induces apoptosis in breast cancer cells through caspase-3 and JNK1 pathways. These mechanisms are related to oxygen radical overproduction during metabolic activation of tamoxifen [1]. Although the beneficial effects of tamoxifen are based on its effects as an estrogen antagonist in breast tissue, its use is associated with proestrogenic effects in other tissues, including increases in hepatic coagulation factor synthesis $[2,3]$. Adverse outcomes noted with tamoxifen use include an increased incidence of cardiovascular events for example, deep vein thrombosis (DVT), pulmonary embolism, and stroke. Although the exact mechanism(s) for its prothrombotic effect is unknown, studies demonstrate that platelets treated with active metabolites of tamoxifen increase superoxide release through an NADPH oxidasedependent mechanism and are associated with increased intracellular free calcium, leading to their activation $[4,5]$. Contrary to this commonly noted observation, we report a case where, surprisingly, tamoxifen treatment was associated with decreased platelet activation and bleeding.

\section{Case Report}

We evaluated a 45-year-old female who presented with a 2-week history of easy bruising 4-6 weeks after taking only tamoxifen $(20 \mathrm{mg} /$ day). She was stage II (T1N1M0) left breast adenocarcinoma patient treated with partial mastectomy and axillary lymph node dissection followed by chemotherapy with adriamycin, cytoxan, and paclitaxel followed by irradiation. The patient's bruisability was associated with $45 \mathrm{~min}$ of bleeding after tooth extraction. She had no previous bleeding history that includes surgeries for a decompressive laminectomy and fusion, an iliac crest bone graft, bilateral breast reduction, ankle surgery, and the mastectomy. The patient denied oral ingestion of aspirin, nonsteroidal anti-inflammatory drugs (NSAIDs), or selective serotonin reuptake inhibitors (SSRIs), which are known to affect platelet function. Physical examination showed one large bluish-purple ecchymosis on the left leg and few smaller resolving bruises on both lower limbs. In the laboratory she had a normal complete blood count, renal and hepatic function, PT, APTT, fibrinogen, factor XIII, von Willebrand antigen and multimers, ristocetin cofactor assay, ristocetininduced platelet aggregation, and no platelet-associated immunoglobulin. Platelet function studies revealed normal 
TABLE 1: Platelet aggregation and activation assays.

\begin{tabular}{|c|c|c|c|}
\hline & $12 / 08$ & $3 / 09$ & Normal \\
\hline \multicolumn{4}{|c|}{ Platelet aggregation } \\
\hline \multicolumn{4}{|l|}{$\mathrm{ADP}$} \\
\hline Percentage of aggregation & 78 & 61 & $70-100 \% \max$ \\
\hline ATP release & 0.85 & 0.23 & $0.2-1.6 \mathrm{nM}$ \\
\hline \multicolumn{4}{|l|}{ AA } \\
\hline Percentage of aggregation & 76 & 71 & $66-100 \% \max$ \\
\hline ATP release & 0.68 & 0.29 & $0-1.3 \mathrm{nM}$ \\
\hline \multicolumn{4}{|l|}{ Collagen } \\
\hline Percentage of aggregation & 87 & 74 & $70-100 \% \max$ \\
\hline ATP release & 1.02 & 0.53 & $0.4-1.9 \mathrm{nM}$ \\
\hline \multicolumn{4}{|l|}{ Epinephrine } \\
\hline Percentage of aggregation & 80 & 66 & $67-95 \% \max$ \\
\hline ATP release & 1.02 & 0.54 & $0.3-1.7 \mathrm{nM}$ \\
\hline \multicolumn{4}{|c|}{ Platelet activation } \\
\hline Percentage of mepacrine uptake & 33 & 63 & $54-84 \%$ \\
\hline Percentage of mepacrine release & 82 & 79 & $58-93 \%$ \\
\hline
\end{tabular}

ADP: adenosine diphosphate; AA: arachidonic acid.

ADP-, epinephrine-, arachidonic acid-, and collageninduced platelet aggregation and ATP release (Table 1). However, the patient had reduced mepacrine uptake but with normal release, suggesting an acquired storage pool disorder $[6,7]$. It is recognized that patients with acquired storage pool disorders can have normal platelet aggregation and secretion studies [8,9]. After tamoxifen was stopped, the patient's bruising ameliorated. Repeat platelet function studies performed 3 months later revealed normal platelet function studies and full correction of the mepacrine uptake defect (Table 1).

\section{Discussion}

These studies suggest the development of an acquired storage pool disorder with a reversible reduction in dense granule uptake associated with tamoxifen therapy. Platelet storage pool disorders arise from decreased platelet granule content of dense, alpha, or combined granules that are associated with reduced platelet function and bleeding. Dense granules contain 5-hydroxytryptamine (serotonin) and ATP/ADP [8, 9]. Mepacrine is a dense granule marker used for flow cytometry [10]. Quantitative reduction of any dense granule constituent is sufficient for the diagnosis of a storage pool disorder [8-10]. Although inherited platelet storage pool defects are rare, acquired platelet storage pool disorders are a more common occurrence seen in conjunction with autoimmune disorders such as systemic lupus erythematosus, cardiovascular bypass, and hematological disorders such as hairy cell leukemia, myelodysplasia, and myeloproliferative disorders [11-13]. In vivo platelet activation followed by reduction in circulating granule content release leads to a state of partial activation of platelets with acquired platelet dysfunction. Common entities associated with an acquired storage pool disorder include cardiac bypass with contact with the membrane oxygenator and immune complexes binding to platelets. Patients typically present with "platelet-type" hemorrhagic symptoms and signs such as mucosal membrane bleeding or prolonged bleeding postoperatively. Our report is the first to our knowledge to implicate an oral anticancer therapy producing an acquired platelet storage pool disorder.

Tamoxifen and Raloxifene, another selective estrogen receptor modulator (SERM), have been associated with increased platelet $\mathrm{NO}$ and reduced peroxynitrite leading to reduced platelet function $[14,15]$. In experimental studies, tamoxifen treatment in micromolar concentrations inhibits all platelet functions with collagen activation being most sensitive, prolongs the bleeding time, and delays arterial thrombosis in vivo $[16,17]$. Although the precise mecha$\operatorname{nism}(\mathrm{s})$ contributing to an acquired storage pool disorder is not known, the ability of tamoxifen treatment to inhibit $\mathrm{Ca}^{2+}$ mobilization and IP3 phosphorylation suggest that there is reduced platelet activation leading to granule release $[16,17]$.

At the therapeutic dose of $20-40 \mathrm{mg} /$ day, a concentration of $1 \mu \mathrm{M}$ of tamoxifen is achieved in the blood. Significant inhibition of platelet function, as noted by Nanetti et al., occurred only at tamoxifen levels of $5 \mu \mathrm{M}$ and above [14]. It is possible that our patient may be more sensitive to the antiplatelet effects of tamoxifen at therapeutic drug levels. Alternatively, the pharmacokinetics in this patient may have resulted in higher than usual levels of tamoxifen leading to the platelet dysfunction. Considering the effect of tamoxifen on platelet function, it is surprising that the observations noted in the present patient have not been previously appreciated.

\section{References}

[1] S. Mandlekar, R. Yu, T. H. Tan, and A. N. T. Kong, "Activation of caspase-3 and c-Jun NH2-terminal kinase-1 signaling 
pathways in tamoxifen-induced apoptosis of human breast cancer cells," Cancer Research, vol. 60, no. 21, pp. 5995-6000, 2000.

[2] J. Chang, T. J. Powles, S. E. Ashley et al., "The effect of tamoxifen and hormone replacement therapy on serum cholesterol, bone mineral density and coagulation factors in healthy postmenopausal women participating in a randomised, controlled tamoxifen prevention study," Annals of Oncology, vol. 7, no. 7, pp. 671-675, 1996.

[3] K. I. Pritchard, A. H. G. Paterson, N. A. Paul, B. Zee, S. Fine, and J. Pater, "Increased thromboembolic complications with concurrent tamoxifen and chemotherapy in a randomized trial of adjuvant therapy for women with breast cancer," Journal of Clinical Oncology, vol. 14, no. 10, pp. 2731-2737, 1996.

[4] O. Vitseva, D. A. Flockhart, Y. Jin, S. Varghese, and J. E. Freedman, "The effects of tamoxifen and its metabolites on platelet function and release of reactive oxygen intermediates," Journal of Pharmacology and Experimental Therapeutics, vol. 312, no. 3, pp. 1144-1150, 2005.

[5] Y. Dobrydneva, R. V. Weatherman, J. P. Trebley et al., "Tamoxifen stimulates calcium entry into human platelets," Journal of Cardiovascular Pharmacology, vol. 50, no. 4, pp. 380-390, 2007.

[6] J. E. Wall, M. Buijs-Wilts, J. T. Arnold et al., "A flow cytometric assay using mepacrine for study of uptake and release of platelet dense granule contents," British Journal of Haematology, vol. 89, no. 2, pp. 380-385, 1995.

[7] A. S. Ramström, I. H. Fagerberg, and T. L. Lindahl, "A flow cytometric assay for the study of dense granule storage and release in human platelets," Platelets, vol. 10, no. 2-3, pp. 153$158,1999$.

[8] L. Zhou and A. H. Schmaier, "Platelet aggregation testing in platelet-rich plasma: description of procedures with the aim to develop standards in the field," American Journal of Clinical Pathology, vol. 123, no. 2, pp. 172-183, 2005.

[9] H. K. Nieuwenhuis, J.-W. N. Akkerman, and J. J. Sixma, "Patients with a prolonged bleeding time and normal aggregation tests may have storage pool deficiency: studies on one hundred six patients," Blood, vol. 70, no. 3, pp. 620-623, 1987.

[10] H. P. Lorez, M. Da Prada, F. Rendu, and A. Pletscher, "Mepacrine, a tool for investigating the 5 hydroxytryptamine organelles of blood platelets by fluorescence microscopy," Journal of Laboratory and Clinical Medicine, vol. 89, no. 1, pp. 200-206, 1977.

[11] J. Zahavi and V. J. Marder, "Acquired "storage pool disease" of platelets associated with circulating antiplatelet antibodies," American Journal of Medicine, vol. 56, no. 6, pp. 883-890, 1974.

[12] H. J. Weiss, M. H. Rosove, B. A. Lages, and K. L. Kaplan, "Acquired storage pool deficiency with increased plateletassociated IgG. Report of five cases," American Journal of Medicine, vol. 69, no. 5, pp. 711-717, 1980.

[13] T. W. Malpass, B. Savage, S. R. Hanson, S. J. Slichter, and L. A. Harker, "Correlation between prolonged bleeding time and depletion of platelet dense granule ADP in patients with myelodysplastic and myeloproliferative disorders," Journal of Laboratory and Clinical Medicine, vol. 103, no. 6, pp. 894-904, 1984.

[14] L. Nanetti, A. Camilletti, C. M. Francucci et al., "Role of raloxifene on platelet metabolism and plasma lipids," European Journal of Clinical Investigation, vol. 38, no. 2, pp. 117-125, 2008.

[15] V. P. Shah, H. A. Chegini, S. R. Visneski, R. V. Weatherman, P. F. Blackmore, and Y. Dobrydneva, "Tamoxifen promotes superoxide production in platelets by activation of PI3-Kinase and NADPH oxidase pathways," Thrombosis Research, vol. 129, pp. 36-42, 2012.

[16] M. K. Nayak, S. K. Singh, A. Roy, V. Prakash, A. Kumar, and D. Dash, "Anti-thrombotic effects of selective estrogen receptor modulator tamoxifen," Thromb Haemost, vol. 106, pp. 624635, 2011.

[17] Y. Chang, J. J. Lee, W. F. Chen, D. S. Chou, S. Y. Huang, and J. R. Sheu, "A novel role for tamoxifen in the inhibition of human platelets," Translational Research, vol. 157, no. 2, pp. 81-91, 2011. 


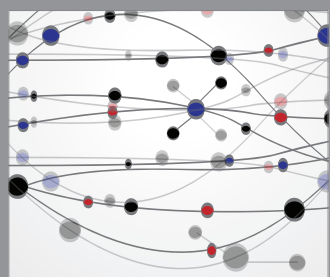

The Scientific World Journal
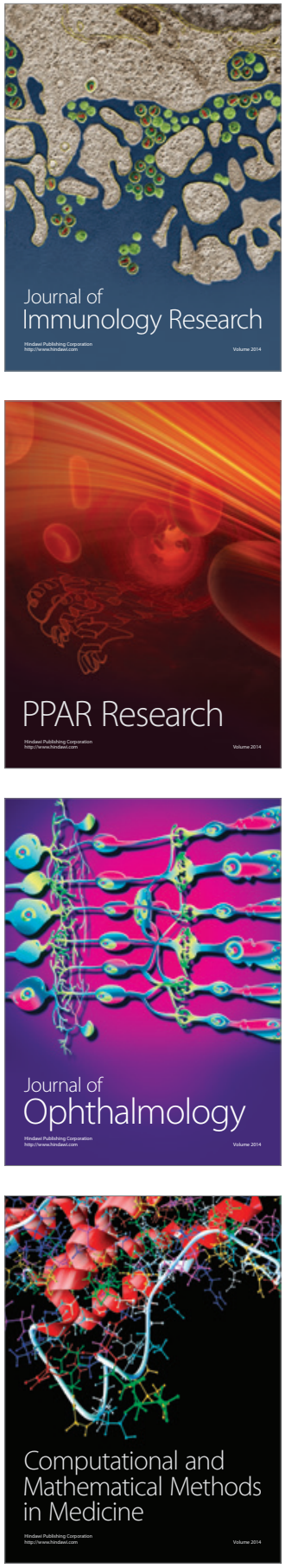

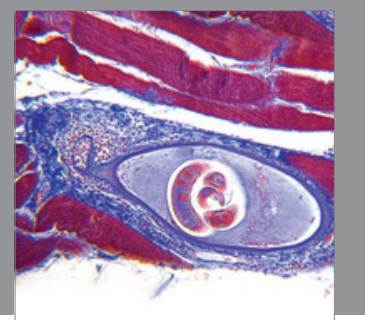

Gastroenterology

Research and Practice
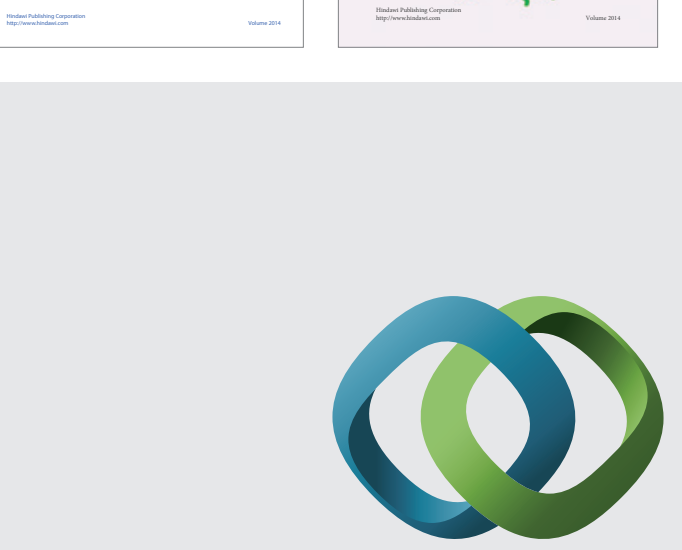

\section{Hindawi}

Submit your manuscripts at

http://www.hindawi.com
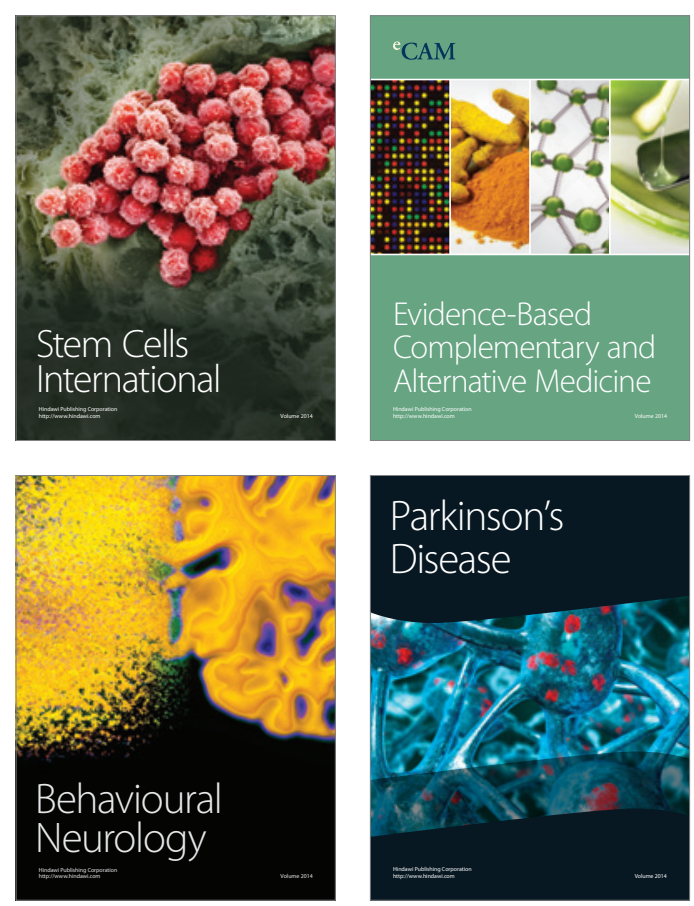

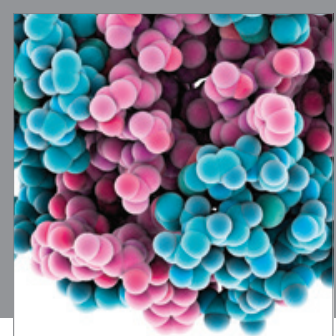

Journal of
Diabetes Research

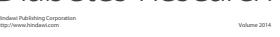

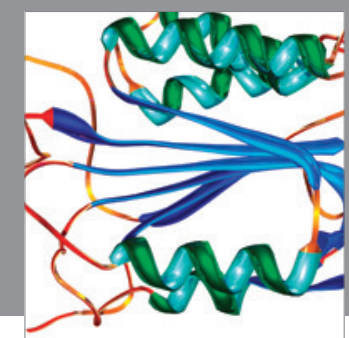

Disease Markers
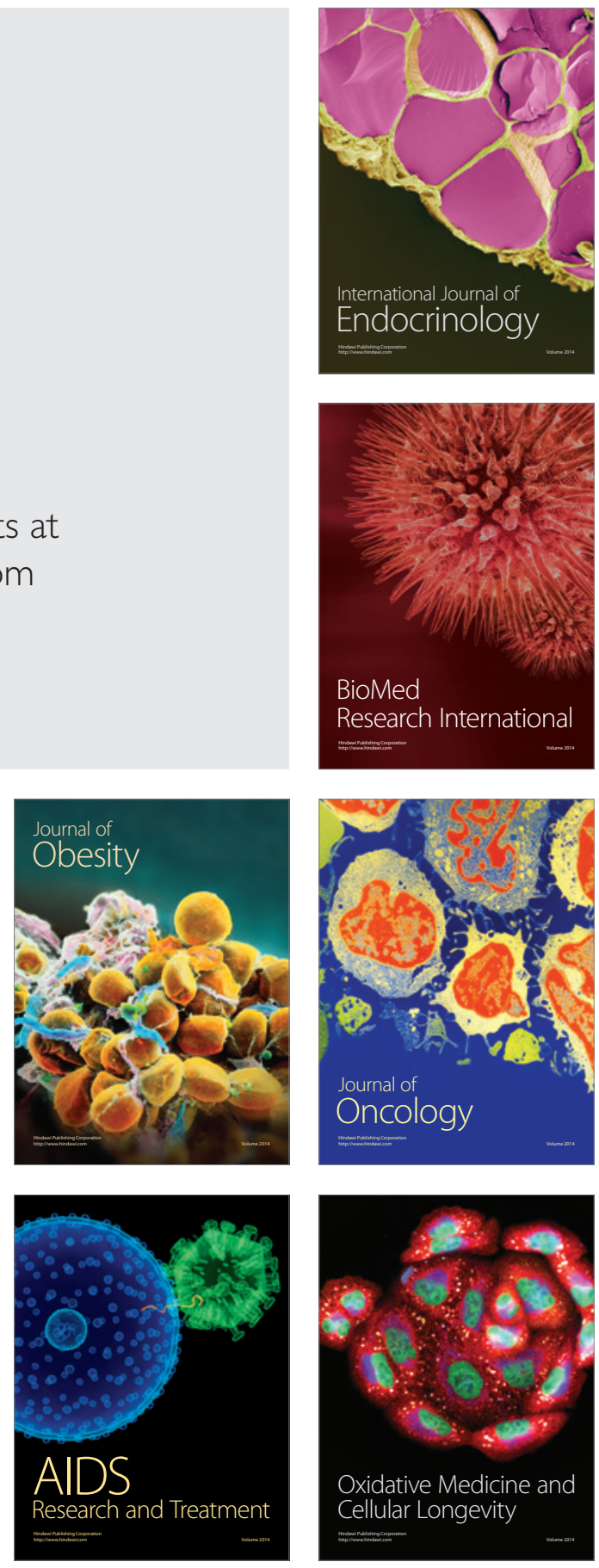